\title{
SPECTRAL ENERGY TRANSFER, GENERATION OF ZONAL FLOWS AND THEIR ROLE IN CONFINEMENT TRANSITIONS
}

\author{
MICHAEL G. SHATS, $*$ HUA XIA, HORST PUNZMANN, and WAYNE M. SOLOMON $\dagger$ \\ Research School of Physical Sciences and Engineering, The Australian National University, Canberra, ACT 0200, Australia
}

Received November 19, 2003

Accepted for Publication April 1, 2004

An overview of recent results related to the physics of turbulent structure generation and their interactions in the $\mathrm{H}-1$ heliac is presented. In particular, the role of zonal flows, or time-varying shear radial electric fields, in anomalous transport and confinement transitions is investigated. It is shown that large-scale coherent structures, including zonal flows, are generated through an inverse energy cascade from the unstable spectral range. Once developed, zonal flows affect other turbulent structures and reduce the particle transport driven by them. The phase randomization of coherent structures by zonal flows is shown to be responsible for reducing the anomalous transport. Zonal flows are also shown to act as precursors during spontaneous low-to-high transitions in $\mathrm{H}-1$.

KEYWORDS: turbulent structures, confinement transitions, toroidal plasma

\section{INTRODUCTION}

The physics of confinement transitions has been a focus of the magnetic fusion community in the last 20 years, since the discovery of the low-to-high (L-H) transitions in the ASDEX tokamak. ${ }^{1}$ Understanding phenomena related to these transitions requires detailed experimental and theoretical studies into the spatial structure, dynamics, and interplay between turbulence and sheared $\mathbf{E} \times \mathbf{B}$ flows in the plasma. These flows, both mean and time varying, play a crucial role in the transition dynamics and turbulence suppression. ${ }^{2}$ However,

\footnotetext{
*E-mail: Michael.Shats@anu.edu.au

$\dagger$ Current address: Princeton University, Princeton Plasma Physics Laboratory.
}

flow generation mechanisms and interaction with turbulent fluctuations still need to be clarified. Among them are the generation of zonal flows, or time-varying shear flows; their interaction with other turbulent structures; their effect on anomalous transport; and their role in bifurcation dynamics. These issues have been studied in the H-1 heliac, and some recent results are reviewed in this paper.

We report results obtained in the $\mathrm{H}-1$ toroidal heliac, a three-field-period helical axis stellarator, which has a major radius of $R=1 \mathrm{~m}$ and a mean minor radius of about $\langle a\rangle \approx 0.2 \mathrm{~m}$. Experiments are performed at low magnetic field in the range of $B=0.05$ to $0.15 \mathrm{~T}$ in the plasma produced by $\sim 60 \mathrm{~kW}$ of radio-frequency waves at $7 \mathrm{MHz}$. Plasma parameters are as follows: $n_{e} \sim 1 \times 10^{18} \mathrm{~m}^{-3}$, $T_{e} \sim 10 \mathrm{eV}, T_{i} \sim 40 \mathrm{eV}$ in argon at filling pressures of $\sim 3 \times 10^{-5}$ torr. Such plasma parameters make the H-1 plasma dimensionally similar to the edge plasma of larger toroidal experiments. This similarity and its implication on the diagnostic opportunities in studying transport barriers are addressed in Sec. IV.

Section II presents new results on the spectral energy transfer in the turbulence spectra. It is shown that inverse cascades are responsible for the generation of large structures in the plasma. The same mechanisms are responsible for the generation of zonal flows. Zonal flows subsequently modify the particle transport driven by other turbulent structures. The details of this effect are described in Sec. III. Section IV presents new experimental results on the temporal evolution of the L-H transition and discusses the role played by zonal flows.

\section{GENERATION OF TURBULENT STRUCTURES VIA INVERSE SPECTRAL ENERGY CASCADES}

Turbulence in toroidal plasmas is characterized by broadband spectra extending over a broad range of wave numbers and frequencies. These spectra often have maxima at the longest observable scale (the smallest wave 
number) and at low frequencies. Instabilities, which drive turbulence, are initially localized in a narrow spectral range, typically not coinciding with the maxima of the spectra of the turbulence. As unstable fluctuations develop, nonlinear mechanisms lead to spectral broadening of the unstable range. Among such mechanisms are threewave interactions, which lead to the transfer of the spectral energy toward both lower and higher wave numbers. The former spectral energy transfer is often referred to as an inverse energy cascade, while the latter is called a forward cascade. The inverse cascade has long been suspected to be the main mechanism in driving large-scale structures in toroidal plasma; however, experimental verification of this hypothesis appeared to be difficult. One of the main difficulties might have been the inadequacy of the single-field description of turbulence (HasegawaMima type model) in the high-temperature toroidal plasma. This problem can be avoided under certain plasma conditions in the $\mathrm{H}-1$ heliac, as explained in Ref. 3, where the single-field description has been justified.

The first experimental evidence that the inverse energy cascade in turbulent spectra is linked to the generation of large-scale structures in the toroidal plasma is presented in Ref. 3. Such a condensation of the spectral energy was theoretically predicted by Hasegawa et al. ${ }^{4}$ Our results on the spectral power transfer based on the extended Ritz model are correlated with this prediction and also agree with the expected shape of the turbulent spectra.

A technique for a quantitative estimation of the energy cascading between waves in turbulent plasma was developed by Ritz et al. ${ }^{5}$ and later extended by Kim et al. ${ }^{6}$ This technique is based on an analysis of the wave kinetic equation describing the evolution of the spectral power of the $k^{\prime}$ th spectral component, $P_{k}=\phi_{k}(t) \phi_{k}^{*}(t)$ (where $\phi_{k}$ is the fluctuating electrostatic potential and asterisk denotes complex conjugate):

$$
\frac{\partial P_{k}}{\partial t} \approx \gamma_{k} P_{k}+\sum_{k=k_{1}+k_{2}} T_{k}\left(k_{1}, k_{2}\right)
$$

where $T_{k}\left(k_{1}, k_{2}\right)$ is the nonlinear power transfer function and $\gamma_{k}$ is the linear growth (or damping) rate. The nonlinear energy transfer function (NETF) is then determined as

$$
W_{N L}^{k} \approx\left(1+k_{\perp}^{2}\right) \sum_{k=k_{1}+k_{2}} T_{k}\left(k_{1}, k_{2}\right) .
$$

Crossley et al. ${ }^{7}$ have proposed the "amplitude correlation" technique, which complements the power transfer function method ${ }^{5}$ and allows a better spectral resolution to be achieved, as described in Refs. 3 and 8 .

Results of the nonlinear energy transfer computations are presented in Fig. 1. First, we compare power spectra of the potential fluctuations and their NETF in low $(\mathrm{L})$ and high $(\mathrm{H})$ confinement modes. One of the differences between these two plasma states is the strongly sheared radial electric field in $\mathrm{H}$ mode, which is correlated with a substantial reduction in the fluctuation level in this mode. The fluctuation spectrum in $\mathrm{L}$ mode in Fig. 1a shows distinct spectral features in the lowfrequency range, $f<15 \mathrm{kHz}$, and decaying broadband turbulence. Among these coherent features in the spectrum in Fig. 1a is a zonal flow at $f \approx 6 \mathrm{kHz}$, which is discussed below. After the transition to $\mathrm{H}$ mode, fluctuations are suppressed in the entire spectral range except for $f \approx 40 \mathrm{kHz}$. The NETF (Fig. 1b) in L-mode is negative in the spectral range of $f=15$ to $50 \mathrm{kHz}$, while it is positive at lower frequencies. This indicates that waves
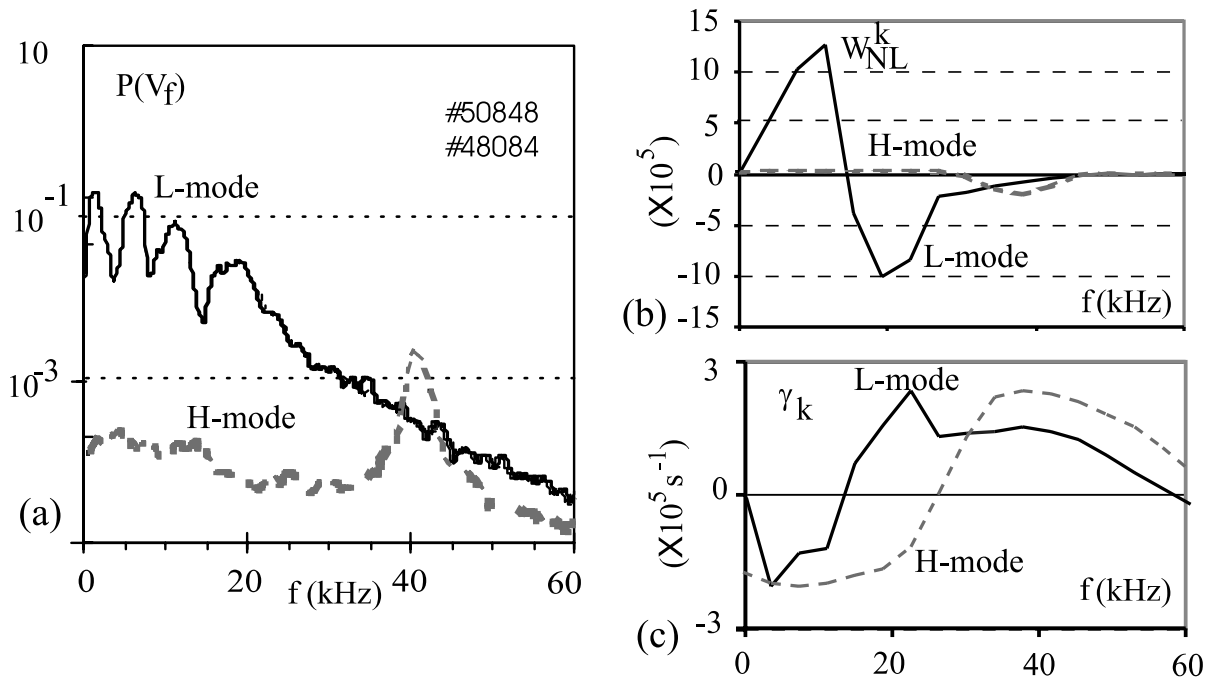

Fig. 1. (a) Power spectra of the floating potential fluctuations; (b) nonlinear energy transfer functions; (c) linear growth rates in $\mathrm{L}$ (solid lines) and $\mathrm{H}$ (dotted lines) confinement modes. Spectra in (a) are computed with a frequency resolution of $0.5 \mathrm{kHz}$, while in (b) and (c) the resolution is $4 \mathrm{kHz}$ because of statistical averaging. 
in the higher-frequency range (higher $k$, as discussed in Ref. 3) give away spectral energy while the lowerfrequency spectral features on average receive energy through a three-wave interaction process. Such an inverse cascade of energy may be responsible for the generation of large structures seen as coherent features in the L-mode spectrum. We define the nonlinear energy flow into the low-frequency range as $W_{N L}^{i n}=\sum_{k}\left(W_{N L}^{k}>0\right)$. This energy is correlated with the generation of the (quasi-) coherent turbulent structures as illustrated in Fig. 2 (Ref. 8), which shows the root-mean-square value of the potential fluctuations (effectively, the spectral power of the low-frequency condensate at $f<15 \mathrm{kHz}$ ) compared with $W_{N L}^{i n}$. When the nonlinear energy transfer into the spectral condensate becomes sufficiently high, zonal flows (largest anisotropic structures ${ }^{9}$ ) are observed. Spectral power in the range of $f<15 \mathrm{kHz}$ increases with the increase in the total nonlinear energy flow into this range, $W_{N L}^{i n}$. In $\mathrm{H}$ mode, $W_{N L}^{i n}$ is low, and no low-frequency structures are observed. In L-mode, poloidally isotropic coherent structures $\left(k_{r} \approx k_{\theta}\right)$ develop in a somewhat intermediate range of $W_{N L}^{i n}$. Finally, at highest $W_{N L}^{i n}$, L-mode spectra show zonal flows. More details on zonal flows in $\mathrm{H}-1$ are given at the end of this section. At this point, we state that the generation of zonal flows in L-mode is correlated with the increased energy input into the condensate through three-wave interactions.

The spectral energy is nonlinearly transferred into large-scale coherent structures from the spectral range of $f=15$ to $50 \mathrm{kHz}$. The linear growth rate is determined from Eq. (1) and is shown in Fig. 1c. The linear growth rate exhibits a peak at $f \sim 25 \mathrm{kHz}$ in the L mode and at $f \sim 35$ to $40 \mathrm{kHz}$ in $\mathrm{H}$ mode. It is natural to assume that this is the spectral range of the underlying linear instability driving fluctuations in this plasma. Our previous work suggested that the unstable fluctuations in $\mathrm{H}-1$ are due to some kind of pressure-gradient driven instability. ${ }^{10}$ In this case, the computed linear growth rate should

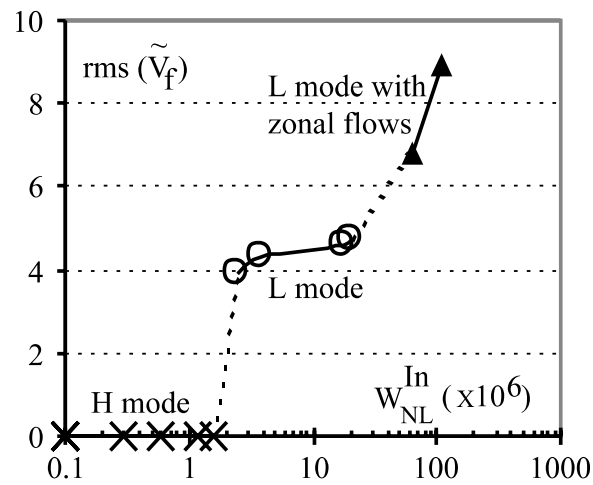

Fig. 2. Spectral power of the low-frequency coherent structures in the range of $f=0$ to $15 \mathrm{kHz}$ compared with nonlinear energy transfer into this frequency range. be correlated with the free-energy reservoir for the instability, namely, with the plasma pressure gradient, which in $\mathrm{H}-1$ is dominated by the ion component since $T_{i}>T_{e}$ (Ref. 11). We estimate the ion pressure gradient $\nabla P_{i}$ from the radial force balance equation:

$$
E_{r} \approx\left(Z_{i} e n\right)^{-1} \nabla P_{i}-V_{\theta i} B_{\phi}+V_{\varphi i} B_{\theta},
$$

in which the velocity terms $V_{\theta i} B_{\phi}$ and $V_{\phi i} B_{\theta}$ are neglected since ion flow velocities in $\mathrm{H}-1$ are small compared with the $\mathbf{E} \times \mathbf{B}$ and the ion diamagnetic drift velocities, as discussed in Ref. 11. The radial electric field and the electron density are measured by Langmuir probes, and the pressure gradient is estimated as $\nabla P_{i} \approx$ $E_{r} Z_{i}$ en. Figure 3 shows the linear growth rate $\gamma_{k}$ of waves in the range of $f=25$ to $35 \mathrm{kHz}$ compared with the pressure gradient $\nabla P_{i}$. The observed linear relation between $\gamma_{k}$ and $\nabla P_{i}$ supports our expectations that the fluctuations in the unstable range of $f=15$ to $50 \mathrm{kHz}$ in $\mathrm{L}$ mode are driven by a pressure-gradient instability. ${ }^{8} \mathrm{We}$ may speculate that initially the instability develops in a narrow spectral region that gradually broadens by nonlinear mechanisms until the spectral power accumulates in the lower-frequency region. The inverse spectral energy transfer is probably responsible for driving large coherent structures, including zonal flows. Generation of zonal flows requires higher levels of nonlinear energy transfer compared with other structures, as seen in Fig. 2.

Zonal flows are potential structures whose radial $k_{r}$ and poloidal $k_{\theta}$ wave numbers are such that $k_{r} \gg k_{\theta} \approx 0$. In other words, zonal flows are poloidally extended structures. They can also be considered as time-varying shear $\mathbf{E} \times \mathbf{B}$ flows. These $\widetilde{\mathbf{E}} \times \mathbf{B}$ shear flows can be generated by turbulent Reynolds stress ${ }^{12}$ or through other mechanisms in which fluctuations drive radial currents, thus contributing to the poloidal momentum balance equation:

$$
\partial V_{\theta} / \partial t=-\partial / \partial r\left(\widetilde{V}_{r} \widetilde{V}_{\theta}\right)-J_{r} B_{\theta} /\left(m_{i} n\right)-\mu V_{\theta},
$$

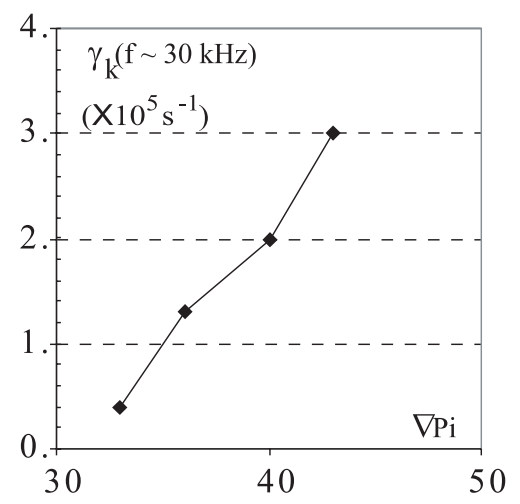

Fig. 3. Computed linear growth rate of the fluctuations in the range of $f=25$ to $35 \mathrm{kHz}$ compared with the ion pressure gradient in L-mode plasma discharges. 
where

$$
\begin{aligned}
V_{\theta}, V_{r} & =\text { poloidal and radial flow velocities } \\
B_{\phi} & =\text { toroidal magnetic field } \\
\mu & =\text { damping rate of the mean flow. }
\end{aligned}
$$

A radial current $J_{r}$ can result from the nonambipolarity of the fluctuation-driven transport, $J_{r}^{f l}=e\left\langle\tilde{n}\left(\widetilde{V}_{e r}-\widetilde{V}_{i r}\right)\right\rangle$, if the electron and ion radial fluctuating velocities are different, $\widetilde{V}_{e r} \neq \widetilde{V}_{i r}$, for example, because of the finite ion Larmor radius effect. Reference 13 reports that the fluctuations in the $\mathrm{H}-1$ heliac do generate radial currents so that the second term in the right side of Eq. (4) appears to be larger than the turbulent Reynolds stress (first term). Thus, the nonambipolarity of the fluctuation-driven particle fluxes could also lead to the generation of a timevarying radial electric field.

The existence of nonambipolar fluctuation-driven particle transport in H-1 allows a slightly different interpretation of the zonal flow generation mechanism. This alternative interpretation is particularly useful in situations where, as in $\mathrm{H}-1$, the ion velocity terms in the radial force balance, Eq. (3), are small compared with $E_{r}$ and $\left(Z_{i} \text { en }\right)^{-1} \nabla P_{i}$ as mentioned above. We rewrite the Poisson's equation to separate radial currents in the plasma according to their dependence on $E_{r}$ :

$$
\begin{aligned}
\varepsilon_{0} \varepsilon_{\perp} \frac{\partial E_{r}}{\partial t} & =q \sum_{k}\left(\Gamma_{e}^{k}-\Gamma_{i}^{k}\right) \\
& =J_{r}^{f l}\left(E_{r}\right)+J_{r}\left(E_{r}\right)+J_{r}(\text { others }),
\end{aligned}
$$

where $J_{r}^{f l}\left(E_{r}\right)$ is the fraction of the radial current driven by the nonambipolar fluctuation-induced particle transport and $J_{r}\left(E_{r}\right)$ relates to other currents in the plasma that are functions of $E_{r}$ (e.g., neoclassical return current). Equation (5) appears to be structurally similar to Eq. (4) used to describe the mode coupling during zonal flow generation (see Ref. 14). The $J_{r}^{f l}\left(E_{r}\right)$ term can be considered as the growth term, while $J_{r}\left(E_{r}\right)$ is analogous to the damping $\mu V_{\theta}$ term in the poloidal momentum balance, Eq. (4).

It should be noted that the above interpretation of the fluctuation-driven radial current being a driving force for zonal flow generation is in agreement with the original interpretation of the Reynolds stress as a radial current, as discussed in Ref. 12.

Zonal flows are usually considered as purely potential structures; in other words, oscillations at the zonal flow frequency are expected only in the potential field with very little or no response in the density fluctuations, as noted in Ref. 15. This, however, may depend on the coupling between $\widetilde{E}_{r}$, the ion pressure, and the ion flows according to Eq. (3). If $E_{r} \approx\left(Z_{i} e n\right)^{-1} \nabla P_{i}$, as is observed in H-1 (Ref. 11), then the zonal-flow-driven oscillations in $\widetilde{E}_{r}$ may be balanced by the fluctuations in the ion pressure term: $\left(Z_{i} e \tilde{n}\right)^{-1} \nabla\left(\tilde{n} T_{i}\right)$. In this case, zonal flows may be observed in the density field as well as in the potential field. This is the case in H-1 (Ref. 3) and could also be the case in DIII-D (Ref. 16), where the zonal flow signatures were observed in the density fluctuations.

The identification of zonal flows as poloidally extended structures in the $\mathrm{H}-1$ heliac is described in (Ref. 9). These structures play an important role in locally controlling the anomalous particle transport driven by the finite- $k_{\theta}$ structures, as shown in Sec. II.

Another comment should be made with regard to the frequency of zonal flows that are nonlinearly generated by broadband turbulence through three-wave interactions in the inverse energy cascade process discussed above. For obvious reasons, computations of the threewave interactions are performed in the frequency rather than in the wave number domain. In this case, waves participating in the three-wave interactions satisfy frequency matching rules $f=f_{1}-f_{2}$. Strictly speaking, this can be justified only if the linear dispersion [linear $k(\omega)$ dependence $]$ is confirmed experimentally so that the above frequency matching rule also holds for the wave numbers, $k=k_{1}-k_{2}$. It has been demonstrated experimentally ${ }^{8}$ in $\mathrm{H}-1$ that the poloidal wave numbers $k_{\theta}$ do have a linear trend with $f$. One could conclude that a zonal flow characterized by a very small $k_{\theta}$ should have a nearly zero frequency. However, zonal flows observed in $\mathrm{H}-1$ have finite frequencies, as shown in Fig. 1a and in Refs. 3, 9, and 17. For most turbulent spectral features, their poloidal $k_{\theta}$ and radial $k_{r}$ wave number components are much greater than their parallel $k_{\|}$, so that $k \approx \sqrt{k_{\theta}^{2}+k_{r}^{2}}$ and $k_{\theta} \approx k_{r}$. For a zonal flow, $k_{r} \gg k_{\theta} \approx 0$, so that the full wave number is not zero and $k \approx k_{r}$. In this case, the zonal flow may have a finite frequency if $f$ is linearly proportional to $k$ (not to $k_{\theta}$ ).

\section{SUPPRESSION OF FLUCTUATION-DRIVEN TRANSPORT BY ZONAL FLOWS}

Interaction between flows and turbulent structures is the central point of the turbulence self-regulation concept that is being developed with regard to magnetized plasmas. Both mean (time-averaged) and the timevarying shear flows can affect their parent waves and modify the turbulent transport. ${ }^{2}$ Zonal flows have been observed in H-1 (Ref. 9) and in the DIII-D tokamak. ${ }^{18}$

The first experimental results showing that zonal flows greatly reduce turbulent fluxes and also change the statistical properties of large coherent fluctuations through randomization of their phases are presented in Ref. 17. This transport reduction occurs without a reduction in the fluctuation amplitude. The randomization of coherent structure phases occurs through stochastic Doppler shifts in the presence of noncoherent zonal flows.

Zonal flows having $k_{r} \gg k_{\theta} \approx 0$ have been observed in L-mode plasma. These finite-frequency $\left(f_{Z F}=2\right.$ to 6 
$\mathrm{kHz}$ ) zonal flows, which are generated nonlinearly from the broadband turbulence via three-wave interactions in the inverse cascade process, are described in Sec. II.

Zonal flows are not the only structures present in the spectrum of the plasma electrostatic potential in the $\mathrm{L}$ mode. Strong coherent features with $k_{\theta} \approx k_{r} \neq 0$, also generated nonlinearly by the broadband turbulence, produce a substantial particle flux that affects the plasma confinement. ${ }^{10}$

Radial profiles of the electron density and its fluctuations are shown in Fig. 4a. Profiles of the fluctuationdriven flux, $\Gamma_{f l}=\left\langle\tilde{n} \widetilde{V}_{r}\right\rangle=\left\langle\tilde{n} \widetilde{E}_{\theta}\right\rangle / B_{t}\left(B_{t}\right.$ is the toroidal magnetic field), and the relative level of the fluctuations in the radial electric field due to the zonal flow at $f=$ $6 \mathrm{kHz}, R M S\left[\widetilde{E}_{r}(f=6 \mathrm{kHz})\right] /\left\langle E_{r}\right\rangle$ are shown in Fig. $4 \mathrm{~b}$. A remarkable correlation between the maximum in the zonal flow and the minimum in $\Gamma_{f l}$, seen in Fig. $4 b$, is amplified by the fact that the reduction in $\Gamma_{f l}$ is not due to a reduction in the fluctuation level but rather to the reduced correlation between $\tilde{n}_{e}$ and $\widetilde{E}_{\theta}$. The structure of the time-resolved fluxes is illustrated in Figs. $5 \mathrm{a}$ and $5 \mathrm{~b}$ for two radial positions of Fig. 4 , namely, $r / a=0.68$ and $r / a=0.37$, corresponding to the minimum and maximum of the zonal flow intensity. In the absence of the zonal flow, at $r / a=0.68$ (Fig. 5a), the flux is dominated by positive (outward) bursts; however, with the zonal flow, maximum positive and negative bursts nearly balance each other, resulting in a zero time-averaged flux (Fig. 5b). The probability distribution functions of these fluxes are significantly modified; particularly, the skewness (third-order statistical moment) of the flux becomes considerably reduced in the presence of zonal flow.

Zonal flows randomize phases of large coherent structures responsible for large anomalous transport in $\mathrm{H}-1$, as discussed in Ref. 17 . The velocity of the poloidally propagating coherent structures is Doppler shifted by the lowerfrequency zonal flow, so that the fluctuating poloidal electric field is expressed as

$$
\widetilde{E}_{\theta k}=E_{\theta k} \sin \left[\left(\omega_{k}+k_{\theta} \frac{\widetilde{E}_{r}(\Omega)}{B_{t}}\right) t\right],
$$

where $\Omega$ is the frequency of the zonal flow seen as a time-varying radial electric field. In practice, zonal flows are not ideally coherent, and their spectrum has finite width. This Doppler shift randomizes the phase of the propagating potential structures, which leads to substantial modifications in the statistical properties of the fluctuations. ${ }^{17}$ The autocorrelation functions of the fluxes are also modified, as seen in Figs. 5c and 5d. The important result in the context of transport barrier formation is that zonal flows can locally suppress turbulent transport before fluctuations are suppressed. The fluctuation-driven particle flux is defined in the frequency domain as

$$
\Gamma_{f l}=\frac{2}{B} \int_{0}^{\infty}\left[P_{n} P_{E}\right]^{1 / 2} \times\left|\gamma_{n E}\right| \times \cos \left[\alpha_{n E}\right] \times d \omega
$$

where

$$
\begin{aligned}
& 0 \leq\left|\gamma_{n E}\right| \leq 1 \\
& \alpha_{n E}=\begin{array}{l}
\text { coherence and phase shift between } \tilde{n}_{e} \text { and } \\
\widetilde{E}_{\theta}, \text { correspondingly }
\end{array} \\
& P_{n}, P_{E}=\begin{array}{l}
\text { autopower spectral densities of the fluc- } \\
\text { tuations. }
\end{array}
\end{aligned}
$$

This equation shows that the particle flux can be reduced by suppressing the turbulence ( $P_{n}$ and $P_{E}$ reduction), by decorrelating density and potential fluctuations $\left(\gamma_{n E} \rightarrow\right.$ 0 ), or by changing the relative phase $\alpha_{n E}$ between them. Phase randomization in either $\tilde{n}_{e}$ and $\widetilde{E}_{\theta}$, or in both, should result in the decrease of the time-averaged coherence $\gamma_{n E}$ between these fluctuations and may also change the cross phase $\alpha_{n E}$. This would affect the fluctuation-driven particle flux without suppressing the fluctuations, as has been observed in the $\mathrm{H}-1$ experiments. ${ }^{17}$

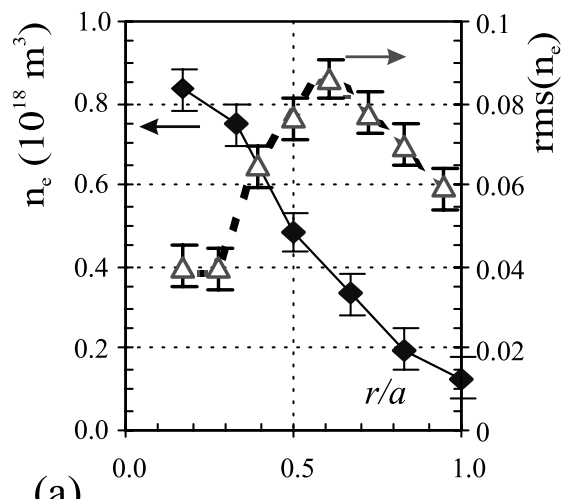

(a)

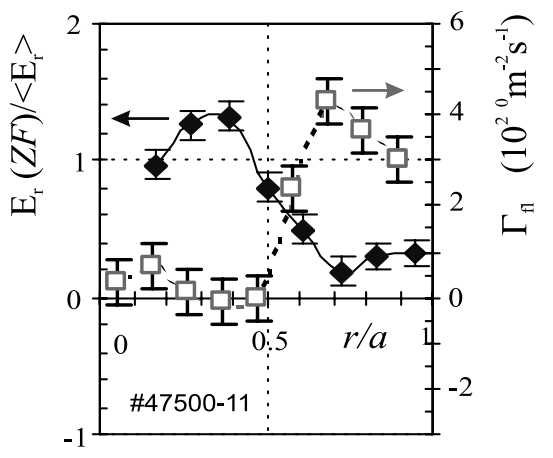

(b)

Fig. 4. Radial profiles of (a) the electron density and the root-mean-square level of its fluctuations and (b) the normalized level of the zonal-flow-driven fluctuations in the radial electric field, $R M S\left[\widetilde{E}_{r}(f=6 \mathrm{kHz})\right] /\left\langle E_{r}\right\rangle$, and of the fluctuation-driven particle flux, $\Gamma_{f l}=\left\langle\tilde{n} \widetilde{E}_{\theta}\right\rangle / B_{t}$. 

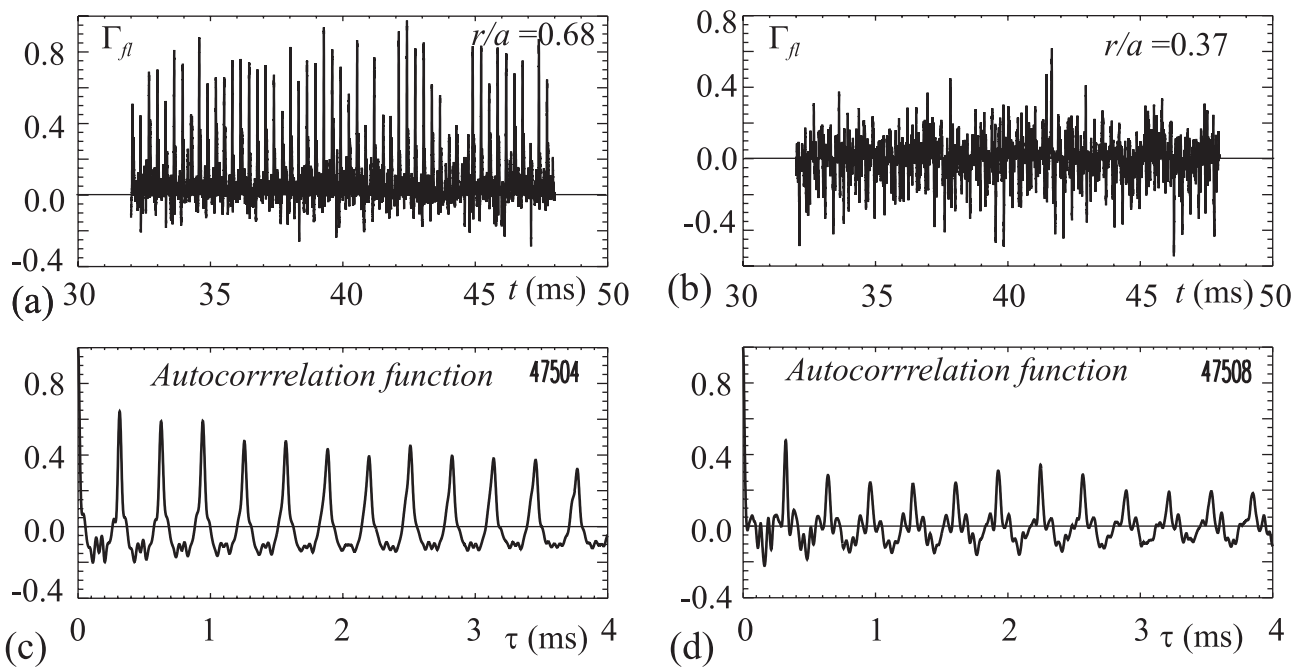

Fig. 5. Time-resolved fluctuation-driven particle fluxes $\Gamma_{f l}$ and their autocorrelation functions measured in the radial regions corresponding to the (a) and (c) minimum and (b) and (d) maximum of the zonal flow at $r / a=0.68$ and at $r / a=0.37$, correspondingly.

The formation of transport barriers due to the phase randomization of the turbulent fluctuations has also recently been found in test-particle simulations of turbulent transport. ${ }^{19}$

\section{THE ROLE OF ZONAL FLOWS IN CONFINEMENT BIFURCATIONS IN H-1}

Sudden changes in the particle confinement observed in the $\mathrm{H}-1$ heliac plasma ${ }^{20,21}$ show many features similar to the L-H transitions in tokamaks, such as a dramatic improvement in the energy and particle confinement, steeper plasma pressure profiles in $\mathrm{H}$ mode, an increase in the radial electric field shear across the transition, and suppression of fluctuations and of the associated anomalous transport.

Similarities between processes in $\mathrm{H}-1$ and in much larger tokamaks can be understood within a concept of dimensionally similar plasmas proposed by Kadomtsev ${ }^{22}$ and developed by Connor and Taylor. ${ }^{23}$ The concept has been used extensively with regard to both tokamaks and stellarators to develop and justify the transport scaling laws capable of predicting plasma confinement in larger fusion-relevant experiments. Plasmas are referred to as dimensionally similar if several essential nondimensional parameters are the same. In light of this concept, a sizable radial region of the low-temperature plasma in $\mathrm{H}-1$ can be considered as dimensionally similar to the narrow region in the tokamak edge plasma where the transport barrier forms during the $\mathrm{L}-\mathrm{H}$ transition.

The pedestal width in $\mathrm{H}-1$ is about $\Delta r=0.06 \mathrm{~m}$ or $\Delta \rho=\Delta r / a \sim 0.3$, while, for example, in the DIII-D tokamak, ${ }^{24}$ this corresponds to $\Delta r \leq 0.015 \mathrm{~m}$ and $\Delta \rho \leq$
0.05. Several nondimensional parameters are similar in the pedestal regions of DIII-D and $\mathrm{H}-1$. The ion gyroradius normalized by the density scale length, $\rho^{*}=\rho_{i} / L_{n}$ (where $L_{n}=n_{e} / \nabla n_{e}$ ), is $\rho^{*} \approx 0.5$ in both machines. The relative collisionality $\nu^{*}$, defined as the ratio of the effective collision rate $\nu_{e i}^{\text {eff }}$ to the bounce frequency $\omega_{b e}$ of the magnetically trapped particles, is about $\nu^{*} \approx 1$ in both H-1 and DIII-D at the pedestal. The ratio of the plasma pressure to the magnetic pressure is $\beta \approx 0.01$ at the pedestal in both experiments. The ranges for all three of the above nondimensional parameters coincide or substantially overlap in both the $\mathrm{H}-1$ confinement region and the DIII-D edge plasmas. In addition, the presence of neutral particles plays an important role at both the DIII-D edge plasma and the partially ionized plasma in the H-1 heliac.

In $\mathrm{H}-1$, the large ion gyroradius in argon discharges at low magnetic fields and relatively high ion temperature $\left(T_{i} \approx 30 \mathrm{eV}>T_{e} \approx 8 \mathrm{eV}\right)$ is responsible for the unusually large value of $\rho^{*}$. As a result, processes that occur in a narrow $(\Delta r \sim 15 \mathrm{~mm})$ radial region in tokamaks are scaled to the confinement region $(\Delta r \sim 60 \mathrm{~mm})$ in $\mathrm{H}-1$, allowing additional diagnostic opportunities for studying the particle transport in $\mathrm{H}-1$ during confinement transitions.

An example of the L-H transition in $\mathrm{H}-1$ under plasma conditions similar to those discussed earlier is shown in Fig. 6a. The electron density doubles across the transition from the $\mathrm{L}$ to $\mathrm{H}$ mode. Before the transition, strong low-frequency oscillations are observed. The $\tilde{n}_{e}$ spectrum is dominated by a strong component at the zonal flow frequency at $\sim 6 \mathrm{kHz}$. This oscillation is present in both the density and the local radial electric field. Potential fluctuations at this frequency are characterized by 


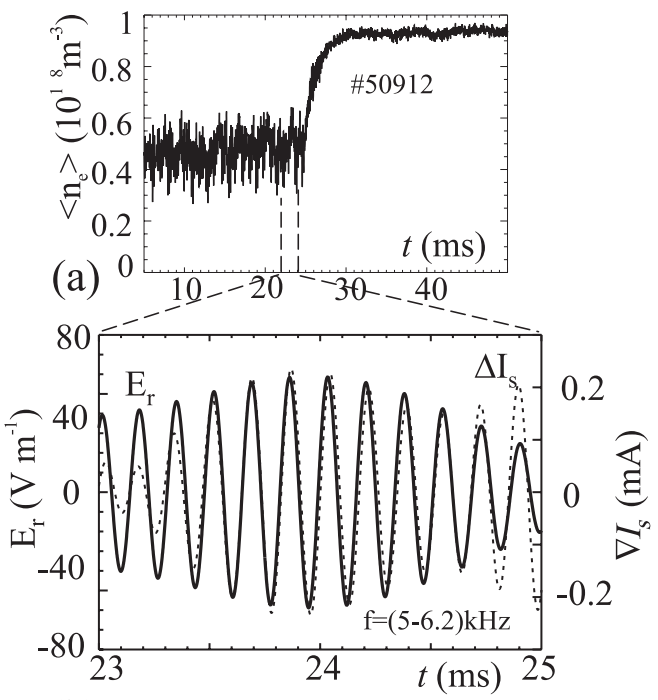

(b)

Fig. 6. Temporal evolution of (a) mean electron density during spontaneous transition from $\mathrm{L}$ to $\mathrm{H}$ mode and (b) oscillations of the radial electric field (solid line) and of the local gradient in the ion saturation current (dotted line) in the zonal flow frequency range of $f=5$ to 6.2 $\mathrm{kHz}$ just before the L-H transition.

$k_{\theta} \approx 0$, which has been measured by two poloidally separated probes, and $\widetilde{E}_{r}$ fluctuations measured by radially separated probes. These local $\widetilde{E}_{r}$ fluctuations at the zonal flow frequency are well correlated with the fluctuations in the local density gradient measured at the same radial location of $r / a \approx 0.5$. This is illustrated in Fig. $6 \mathrm{~b}$, where fluctuations in $E_{r}$ and in the gradient of the ion saturation current $\nabla I_{s}$ (two radially separated probes, $I_{s} \sim n_{e}$ ) oscillate in phase, as one would expect from Eq. (3), considering that the fluctuating ion velocities are small ${ }^{13}$ and assuming that the ion temperature fluctuations are small.

Low-frequency oscillations in the electron density that have poloidal and toroidal mode numbers $m=n=0$ and that precede the $\mathrm{L}-\mathrm{H}$ transitions had been observed ${ }^{25}$ in $\mathrm{H}-1$ though they were not then associated with zonal flows. Colchin et al. ${ }^{26}$ referred to similar oscillations observed in the DIII-D experiment as an intermediate confinement regime (I mode) between $\mathrm{L}$ and $\mathrm{H}$ modes and associated them with self-generated shear flows, though no experimental proof of the zonal flow in I mode was given. Similar dynamics were discovered in a theoretical model ${ }^{27}$ convincingly illustrating the role of the main elements in the transition, namely, zonal flows, mean $\mathbf{E} \times \mathbf{B}$ flows, and the ion pressure gradient. It was demonstrated in Ref. 27 that plasma parameters oscillate at the zonal flow frequency just before the L-H transition, similar to our observation in Fig.6b.

The role of zonal flows in the temporal dynamics of the $\mathrm{L}-\mathrm{H}$ transitions was also confirmed indirectly in
Refs. 14 and 28. The nonlinear coupling between smallscale and larger-scale lower-frequency fluctuations was found to increase transiently before and during spontaneous L-H transitions in the DIII-D tokamak. ${ }^{28}$ It was argued that such an increase was consistent with expectations for a transient Reynolds stress-driven zonal flow triggering the L-H transition.

Our results confirm for the first time that lowfrequency oscillations in the plasma density and in the radial electric field, which precede the L-H transitions, are due to the development of strong zonal flows in the plasma. It should be noted that the role of zonal flow as an important factor in the $\mathrm{L}-\mathrm{H}$ transition dynamics has been confirmed only during spontaneous $\mathrm{L}-\mathrm{H}$ transitions in H-1 and in DIII-D (Refs. 26 and 28). When plasma is sufficiently close to the bifurcation point, zonal flows develop and may act as a transition trigger by increasing the sheared radial electric field above the threshold. If transitions are induced, for example, by external electrode biasing or a sudden increase in heating power, the plasma may skip the zonal flow development stage during its transition to the $\mathrm{H}$ mode.

\section{SUMMARY}

Results presented in this overview can be summarized as follows:

1. Pressure-gradient-driven instability drives fluctuations in the unstable range.

2. Inverse and forward energy cascades generate broadband turbulence and drive large coherent structures that produce anomalous particle transport.

3. Zonal flows are generated at sufficiently high nonlinear energy transfer from smaller-scale to larger-scale fluctuations in turbulent spectra.

4. Zonal flow affects phases of other structures and thus locally suppresses anomalous transport.

5. A mode of confinement, intermediate between low and high (I mode) is observed when zonal flows develop in $\mathrm{L}$ mode prior to spontaneous L-H transitions.

6. Zonal flows in I mode generate oscillations of the plasma parameters at the zonal flow frequency and may act as a trigger for the L-H transition.

\section{REFERENCES}

1. F. WAGNER et al., "Regime of Improved Confinement and High Beta in Neutral-Beam-Heated Divertor Discharges of the ASDEX Tokamak," Phys. Rev. Lett., 49, 1408 (1982).

2. P. W. TERRY, "Suppression of Turbulence and Transport by Sheared Flow," Rev. Mod. Phys., 72, 109 (2000). 
3. H. XIA and M. G. SHATS, "Inverse Energy Cascade Correlated with Turbulent-Structure Generation in Toroidal Plasma," Phys. Rev. Lett., 91, 155001 (2003).

4. A. HASEGAWA, C. G. MACLENNAN, and Y. KODAMA, "Nonlinear Behavior and Turbulence Spectra of Drift Waves and Rossby Waves," Phys. Fluids, 22, 2122 (1979).

5. CH. P. RITZ, E. J. POWERS, and R. D. BENGTSON, "Experimental Measurement of Three-Wave Coupling and Energy Cascading,” Phys. Fluids, B1, 153 (1989).

6. J. S. KIM et al., "Measurements of Nonlinear Energy Transfer in Turbulence in the Tokamak Fusion Test Reactor," Phys. Rev. Lett., 79, 841 (1997).

7. F. J. CROSSLEY et al., "Experimental Study of DriftWave Saturation in Quadrupole Geometry," Plasma Phys. Control. Fusion, 34, 235 (1992).

8. H. XIA and M. G. SHATS, "Spectral Energy Transfer and Generation of Turbulent Structures in Toroidal Plasma," Phys. Plasmas, 11, 561 (2004).

9. M. G. SHATS and W. M. SOLOMON, "Experimental Evidence of Self-Regulation of Fluctuations by Time-Varying Flows," Phys. Rev. Lett., 88, 045001 (2002).

10. M. G. SHATS, "Effect of the Radial Electric Field on the Fluctuation-Produced Transport in the H-1 Heliac," Plasma Phys. Control. Fusion, 41, 1357 (1999).

11. M. G. SHATS et al., "Ion Temperature and Plasma Flows in Improved Confinement Mode in the H-1 Heliac," Phys. Plasmas, 4, 3629 (1997).

12. P. H. DIAMOND and Y. B. KIM, "Theory of Mean Poloidal Flow Generation by Turbulence," Phys. Fluids, B3, 1626 (1991).

13. W. M. SOLOMON and M. G. SHATS, "Nonambipolarity of Fluctuation Driven Fluxes and Its Effect on the Radial Electric Field," Phys. Rev. Lett., 87, 195003 (2001).

14. G. R. TYNAN et al., "On the Nonlinear Turbulent Dynamics of Shear-Flow Decorrelation and Zonal Flow Generation," Phys. Plasmas, 8, 2691 (2001).
15. T. S. HAHM et al., "Zonal Flow Measurements Concept I," Plasma Phys. Control. Fusion, 42, A205 (2000).

16. S. CODA et al., "Signature of Turbulent Zonal Flows Observed in the DIII-D Tokamak," Phys. Rev. Lett., 86, 4835 (2001).

17. M. G. SHATS et al., "Turbulent Transport Reduction and Randomization of Coherent Fluctuations by Zonal Flows in Toroidal Plasma," Phys. Rev. Lett., 90, 125002 (2003).

18. M. JAKUBOWSKI et al., "Observation of Coherent Sheared Turbulence Flows in the DIII-D Tokamak," Phys. Rev. Lett., 89, 265003 (2002).

19. V. CARBONE et al., "Barriers for Transport in Turbulent Plasmas," Phys. Rev. Lett., 90, 055001 (2003).

20. M. G. SHATS et al., "Improved Particle Confinement Mode in the H-1 Heliac Plasma," Phys. Rev. Lett., 77, 4190 (1996).

21. M. G. SHATS et al., "Thresholds and the Role of the Radial Electric Field in Confinement Bifurcations in the H-1 Heliac," Phys. Plasmas, 5, 2390 (1998).

22. B. B. KADOMTSEV, "Tokamaks and Analysis of Scaling," Fiz. Plazmy, 1, 531 (1975).

23. J. W. CONNOR and J. B. TAYLOR, "Scaling Laws for Plasma Confinement," Nucl. Fusion, 17, 1047 (1977).

24. T. N. CARLSTROM and R. J. GROEBNER, "Study of the Conditions for Spontaneous H (High)-Mode Transitions in DIIID," Phys. Plasmas, 3, 1867 (1996)

25. D. L. RUDAKOV et al., "Dynamic Behavior of the Low to High Confinement Transitions in the H-1 Heliac," Plasma Phys. Control. Fusion, 43, 559 (2001).

26. R. J. COLCHIN et al., "Slow L-H Transitions in DIII-D Plasmas," Phys Rev. Lett., 88, 255002 (2002).

27. E. KIM and P. H. DIAMOND, "Zonal Flows and Transient Dynamics of the L-H Transition," Phys Rev. Lett., 90, 185006 (2003).

28. R. A. MOYER et al., "Increased Nonlinear Coupling Between Turbulence and Low-Frequency Fluctuations at the L-H Transition," Phys. Rev. Lett., 87, 135001 (2001).

Michael G. Shats (MSci, electrical engineering, Kiev Polytechnical Institute, Ukraine, 1981; PhD, General Physics Institute, Russia, 1990) is a senior fellow in the Research School of Physical Sciences and Engineering, The Australian National University, Canberra. His research interests include turbulence in toroidal plasmas, confinement bifurcations, particle and energy transport in plasma, and self-organization in plasma.

Hua Xia (BSci, electronic engineering, Chongqing University, China, 1993; MSci, plasma physics, Southwestern Institute of Physics, China, 1998) is a PhD student in the Research School of Physical Sciences and Engineering, The Australian National University, Canberra. Her research interests include nonlinear spectral energy transfer in plasma turbulence, digital signal analysis, fractal geometry, and chaos. 
Horst Punzmann (BSci, communication engineering, Polytechnic University Regensburg, Germany, 1993) is a PhD student in the Research School of Physical Sciences and Engineering, The Australian National University, Canberra. His research interests include transport studies in toroidal plasma.

Wayne M. Solomon (BSci, 1996, and BSci (Hons), 1997, University of Queensland, Australia; PhD, Australian National University, Canberra, Australia) is a research associate in the Princeton Plasma Physics Laboratory, Princeton University. His research interests include plasma transport, momentum confinement, charge exchange recombination spectroscopy, and experimental tests of neoclassical theory. 